Research Report

\title{
Pyrosequence-Based Typing of Alleles of the HLA-DQB1 Gene
}

BioTechniques 33:166-175 (July 2002)

\author{
S. Ringquist, A.M. Alexander, \\ W.A. Rudert, A. Styche, and \\ M. Trucco \\ University of Pittsburgh School \\ of Medicine, Children's Hospi- \\ tal of Pittsburgh, Pittsburgh, \\ PA, USA
}

\section{ABSTRACT}

DNA typing of alleles of the highly polymorphic HLA-DQB1 gene was performed by Pyrosequencing ${ }^{\mathrm{TM}}$ using purified DNA from the 11th International Histocompatibility Workshop human cell lines and sam ples from the Children's Hospital of Pittsburgh registry of diabetics and their first-degree relatives. Pyrosequencing was optimized for genotyping exon 2 of the HLA-DQB1 gene, but the procedure should be applicable to other HLA loci. The 47 $H L A-D Q B 1$ alleles were readily identifiable, as were the 1128 potential allelic heterozygous combinations. The method required PCR conditions that specifically amplified DQB1 but not the pseudogene, $D Q B 2$. The new method of pyrosequencebased typing can be performed in 96- or 384-well format. The 61 polymorphic residues of $D Q B 1$ exon 2 were identified within four pyrosequencing reactions, obtained by a 70-nucleotide read length in each reaction, in about an hour's time. Allelic combinations of HLA-DQB1 most frequently found in the population of diabetics and their immediate family members were analyzed and successfully compared to typing of the $D Q B 1$ alleles by sequence-specific oligonucleotide probe protocols. Pyrosequence-based typing is compatible with genotyping of allelic combinations expected from heterozygous individuals, resulting in nucleotide resolution of the highly polymorphic HLA system. Using pyrosequencing, more than 750 sample wells can be processed in a working day, resulting in the identification of more than 50000 bases.

\section{INTRODUCTION}

Nucleotide polymorphisms at certain HLA loci correlate with susceptibility to a variety of autoimmune diseases, such as type 1 (insulin-dependent) diabetes (recently reviewed in References 9 and 19). Genetic studies of diabetics and their immediate family members have provided critical information, identifying genetic markers for diabetes $(3,4,6)$, with specific polymorphisms of the HLA-DQB1 locus being the strongest indicators of type 1 diabetes mellitus susceptibility $(2,18,29)$.

Methods for screening the highly polymorphic HLA loci for alleles associated with disease are important for risk evaluation of genetically susceptible individuals. HLA-DQB1 alleles containing a polymorphism encoding the presence of a neutral amino acid residue (e.g., alanine, valine, or serine) at amino acid 57 represent one type 1 diabetes susceptibility marker, while an aspartate at the same position (Asp-57) confers resistance $(2,15,16,27,29)$. However, sequence data, including that of the DQ and DRB alleles, result in haplotype information that can be used to better interpret the degrees of association and susceptibility of various genotypes for type 1 diabetes. More- over, high-resolution typing of the HLA region is required for successful kidney $(11,28)$ and bone marrow $(7,12$, 26) transplantation.

Advances in DNA sequencing technology via automated acquisition and analysis are important additions to the screening and characterization of genetic markers. Pyrosequencing ${ }^{\mathrm{TM}}$ (Pyrosequencing AB, Uppsala, Sweden) methodology represents an im provement to data acquisition, analysis, and allelic identification, including the advantages of real-time data output, resistance to sequencing artifacts associated with analysis by gel electrophoresis, no need for DNA fluorescent labeling, and compatibility with 96- and 384-well microplate formats $(22,25)$.

Pyrosequencing was originally designed for expressed sequence tag sequencing in which short (roughly 10nucleotide) stretches of DNA are analyzed. The pyrosequencing method (22) is a four-enzyme process combining the activities of DNA polymerase, ATP sulfurylase, luciferase, and apyrase. Briefly, the method can be described in four steps: $(i)$ hybridization of sequencing primer to an ssDNA template; (ii) incorporation of a complementary nucleotide and release of pyrophosphate; (iii) use of ATP sulfurylase to convert pyrophosphate and exogenous adenosine $5^{\prime}$ phosphosulfate into ATP, followed by conversion into light via the activity of luciferase; and (iv) degradation of unincorporated dNTP by means of apyrase. Sequencing continues by reiteration of steps 2-4 using the next dNTP to be tested for incorporation into the nascent nucleotide chain. The data 
Table 1. Oligonucleotide Primers Used for PCR and DNA Sequencing

\begin{tabular}{|c|c|c|}
\hline Name & Sequence $\left(5^{\prime} \rightarrow 3^{\prime}\right)$ & Comment \\
\hline \multicolumn{3}{|l|}{ PCR Primers } \\
\hline DQBamp-A & CCTGTGCTACTTCACCAACGG & DQB1 Forward Primer \\
\hline DQBamp-B & Biotin-TEG-CTCGTAGTTGTGTCTGCACAC & DQB1 Reverse Primer \\
\hline SR25 & TTTGACCCCGCAGAGGATTTCGTG & DQB1 Forward Primer \\
\hline SR22 & Biotin-TEG-СTCTCCTCTGCARGATCCC & DQB1 Reverse Primer, Alleles 05/06 \\
\hline SR24 & Biotin-TEG-CTCGCCGCTGCAAGGTCGT & DQB1 Reverse Primer, Alleles 02/03/04 \\
\hline \multicolumn{3}{|c|}{ Model DQB1 Oligonucleotides } \\
\hline SR11 & AGATGTGTCTGGTCACACCCCGCACGCGCTAGCGCGTGCG & Modeled after DQB1*05011 \\
\hline SR14 & AGATGCTTCTGCTCACAAGACGCACGCGCTAGCGCGTGCG & Modeled after DQB1*0201 \\
\hline \multicolumn{3}{|c|}{ Pyrosequencing Primers for HLA-DQB1 Exon 2} \\
\hline SR26 & GACCCCGCAGAGGATTTCG & Nucleotide 10 to 9 \\
\hline SR27 & CAACGGGACGGAGCGCGT & Nucleotide 41 to 58 \\
\hline PSQ1 & ACGGAGCGCGTGCG & Nucleotide 48 to 61 \\
\hline PSQ3 & TCGACAGCGACGTG & Nucleotide 106 to 119 \\
\hline SR30 & GCCGAGTACTGGAACAGCC & Nucleotide 159 to 177 \\
\hline \multicolumn{3}{|c|}{$\begin{array}{l}\text { Primers DQBamp-A and DQBamp-B were designed by the } 11 \text { th International Histocompatibility Workshop (8). Primers } \\
\text { SR25, SR22, and SR24 were designed following the recommendations of Pera et al. (17) but with the additional constraint of } \\
\text { avoiding formation of self-priming } 3 \text { ' secondary structure during pyrosequencing. Primers SR26, SR27, PSQ3, and SR30 are } \\
\text { the minimal primer set required for complete sequencing of DQB1 exon } 2 \text {. }\end{array}$} \\
\hline
\end{tabular}

output is represented graphically by a "pyrogram" consisting of a plot of time versus intensity of light produced (23). Generation of light represents incorporation of a particular nucleotide. In our hands, pyrosequencing has been readily able to read DNA sequences of up to 70 nucleotides. An important advantage of pyrosequencing over other sequencing technologies is that nucleotides are tested individually for incorporation into nascent DNA. The result is that mixed populations of DNA expected from heterozygous individuals are sequenced independently, allowing the resolution of allelic combinations that are impossible to distinguish by conventional sequencing approaches.

Our study was meant to explore the use of pyrosequencing to identify alleles of the various HLA genes in which polymorphic DNA stretches of greater than 250 nucleotides are generally considered. Samples of genomic DNA prepared from human HLA homozygous cell lines developed by the 11 th International Histocompatibility Workshop (8) and samples from the Children's Hospital of Pittsburgh (CHP) Histocompatibility Center study of diabetics and their first-degree relatives listed in the Allegheny County Diabetes Reg- istry $(6,13)$ were selected for genotype analysis. Pyrosequencing was initially used to identify alleles frequently detected with the CHP Histocompatibility Center study population, resulting in high-resolution typing of the highly polymorphic exon 2 region of the HLA-DQB1 gene. Pyrosequencing easily completed a 70-nucleotide read length of each sample in a 96-well sam ple tray within an hour's time, potentially yielding 768 sequence reactions in an 8-h shift or as many as 3072 when performed in a 384-well format, which is sufficient to fully sequence the polymorphic exon 2 region of HLA-DQB1 from roughly 768 individuals. Its accuracy and throughput make pyrosequence-based typing an important addition to the methods available for the identification of known markers in genetic studies and genotyping donors for transplant recipient matching.

\section{MATERIALS AND METHODS}

\section{Materials}

Genomic DNA samples were obtained from donors studied at the CHP Histocompatiblity Center among the di- abetics and their first-degree relatives listed in the Allegheny County Diabetes Registry $(6,13)$ or from lymphoblastoid cell lines made available on the occasion of the 11th International Histocompatibility Workshop that are each homozygous for one of the 12 different, most common, DQB1 alleles (8). Oligonucleotides were purchased from Operon Technologies (Alameda, CA, USA) or synthesized on-site using a model 394 DNA/RNA synthesizer (Applied Biosystems, Foster City, CA, USA) (Table 1). Biotinylated oligonucleotides were purified using HPLC. The pyrosequencer was purchased from Pyrosequencing $\mathrm{AB}$. Reagents for pyrosequencing were obtained from Pyrosequencing $\mathrm{AB}$ and used as recommended. All other chemicals and reagents were of the highest quality obtainable.

\section{Methods}

PCR amplification was performed in $50-\mu \mathrm{L}$ volumes containing Taq buffer (Applied Biosystems), $2 \mathrm{mM} \mathrm{MgCl}_{2}$, $0.2 \mu \mathrm{M}$ each dNTP, $0.2 \mu \mathrm{M}$ forward and biotinylated reverse primers, 1 U Taq DNA polymerase, and $5 \mu \mathrm{L}$ purified genomic DNA (200-500 ng DNA). Amplification included $96^{\circ} \mathrm{C}$ incubation for 
3 min, followed by 32 cycles at $96^{\circ} \mathrm{C}$, $55^{\circ} \mathrm{C}$, and $72^{\circ} \mathrm{C}$ incubation for $30 \mathrm{~s}$ at each step. PCR cycling was followed by a final 5-min incubation at $72^{\circ} \mathrm{C}$. Sam ples were then stored at $-20^{\circ} \mathrm{C}$.

Pyrosequence reactions were performed using 20-40 $\mu \mathrm{L}$ amplified DNA from the PCR mixture. Samples were mixed with $4 \mu \mathrm{L}$ streptavidincoated beads purchased from Amersham Biosciences (Piscataway, NJ, USA) in Binding Buffer (Pyrosequencing $\mathrm{AB}$ ) for 10 min with vigorous mixing. Beads were transferred to a filtration apparatus, and the Binding Buffer was removed by vacuum. DNA attached to the streptavidin beads was denatured by incubation for $1 \mathrm{~min}$ in 50 $\mu \mathrm{L}$ Denaturation Buffer (Pyrosequencing $\mathrm{AB}$ ) and washed twice with $150 \mu \mathrm{L}$ Wash Buffer (Pyrosequencing $\mathrm{AB}$ ). Beads were suspended in $50 \mu \mathrm{L} \mathrm{An-}$ nealing Buffer (Pyrosequencing $\mathrm{AB}$ ), and $40 \mu \mathrm{L}$ were transferred to the 96well PSQ $^{\mathrm{TM}}$ plate (Pyrosequencing $\mathrm{AB})$. The appropriate sequencing primer was added in a volume of $5 \mu \mathrm{L}$ using a $3 \mu \mathrm{M}$ stock solution. Primer annealing was performed by incubating the samples at $80^{\circ} \mathrm{C}$ for $2 \mathrm{~min}$. Samples were allowed to cool for $5 \mathrm{~min}$ at room temperature (usually $24^{\circ} \mathrm{C}$ ) and sequenced by pyrosequencing. A detailed description of the pyrosequencing reaction conditions has been recently published by Gharizadeh et al. (10). Pyrosequence data were quantified and background-corrected using Peak Height Determination Software v1.1 (Pyrosequencing AB).

Pyrosequence-based typing simulation software (unpublished data) was developed in Perl v5.6.1 provided by ActiveState Tool (Vancouver, BC, Canada) under the General Public License. Simulation software was used to generate the expected pyrosequence-associated peak heights by comparing nucleotide dispensation orders used during pyrosequencing to allelic sequence data. Pyrosequence-based typing simulation software also compared simulated peak height data for each possible heterozygous allelic combination to distinguish unique and ambiguous allelic combinations. Documentation for Perl can be found at http://www.perl. com. The pyrosequence-based typing simulation software is available upon request.

\section{RESULTS}

\section{Oligonucleotide-Based Studies}

Pyrosequencing has been applied here to the problem of human leukocyte antigen (HLA) typing. Experiments were designed to evaluate the method's suitability for reading nucleotide sequences modeled after HLA-DQB1 alleles *05011 and *0201 (oligonucleotides SR11 and SR14, respectively) (Table 1). Oligonucleotide sam. ples were designed so that a hairpin structure, formed at the $3^{\prime}$-end, would allow self-priming for DNA sequencing (24). Pyrosequencing was performed on the individual oligonucleotides (Figure 1, A and B). For each sample, the complete 20-nucleotide read length was obtained, consisting of the expected signal intensities for incorporation of up to three identical nucleotides in a row (Figure 1A, dispensation G4). Sequencing of a solution containing an equal mixture of both oligonucleotides (Figure 1C) was performed to simulate the data expected from a heterozygous individual. The resulting pyrogram appeared to reflect correctly the expected signal intensities, as judged from an examination of the single sequence data (Figure 1, A and B). For example, in Figure 1C, oligonucleotide SR11 exhibited sequence-specific signal at dispensations G4, C19, and A21, while oligonucleotide SR14 resulted in specific signals at dispensations $\mathrm{T} 1, \mathrm{C} 3$, G12, and G22. The signal associated with the incorporation of the last nucleotide in the nascent chain was lower than expected, probably the result of poor processivity at this position.

Peak signal intensities from the pyro-

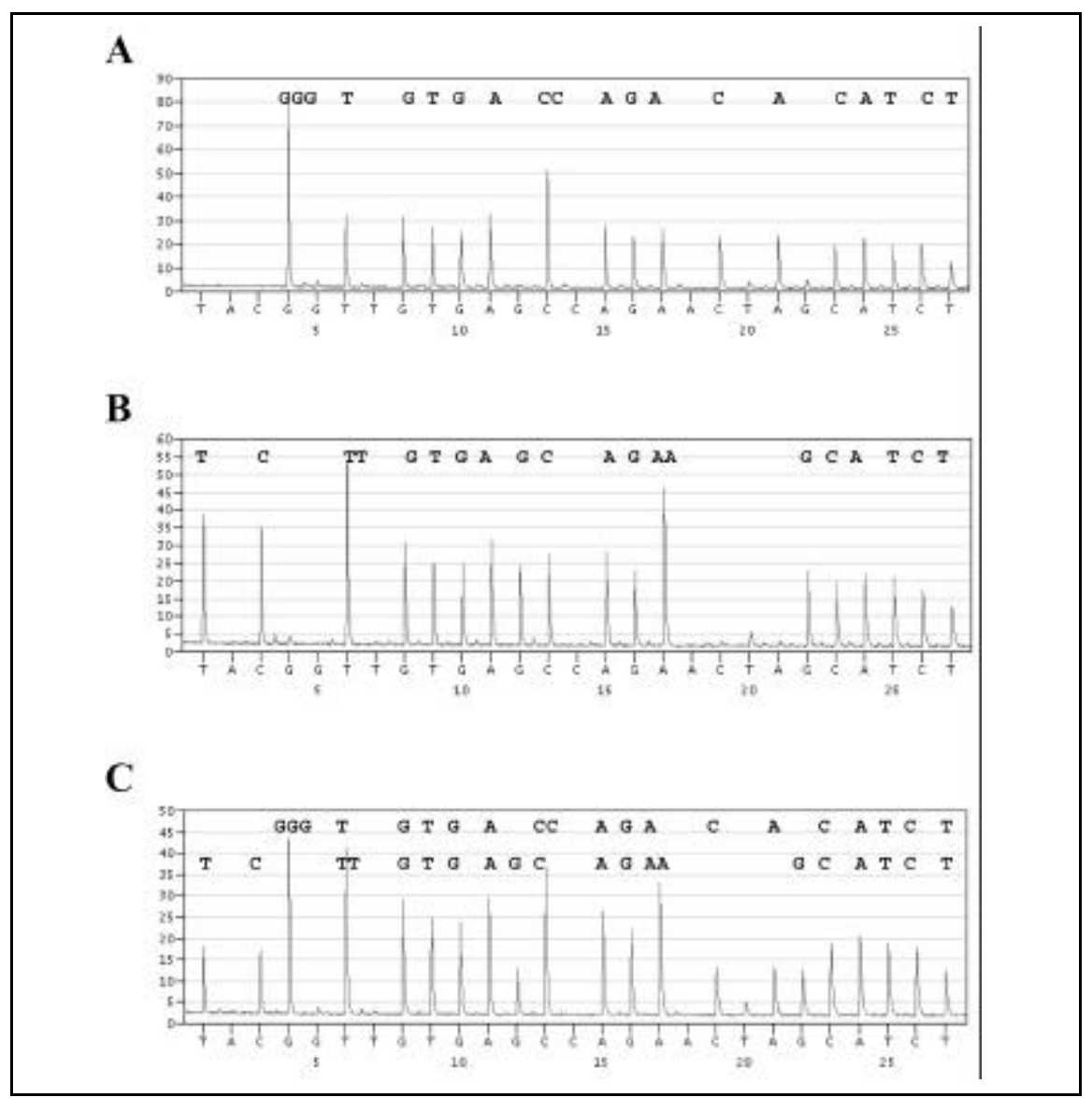

Figure 1. Pyrogram of model DQB1 oligonucleotides SR11 (A), SR14 (B), and the mixture of oligonucleotides SR11 and SR14 (C). Oligonucleotides were present at $0.2 \mu \mathrm{M}$ when used alone and at $0.1 \mu \mathrm{M}$ each when mixed together. Dispensations G4, C19, and A21 are specific for SR11, while T1, C3, G12, and G22 are specific for SR14. The oligonucleotide sequences are indicated in each panel. In panel $\mathrm{C}$, the sequence of SR11 and SR14 are indicated in the top and bottom lines, respectively. Axes are nucleotide dispensation event (x-axis) versus observed signal intensity (y-axis). 
grams in Figure 1 were quantified and background-corrected. Additional analysis was performed by comparison of the observed signal intensities from the mixture of oligonucleotides SR11 and SR14 (Figure 1C) with the calculated signal intensities obtained from sequencing these oligonucleotides individually (Figure 1, A and B). As illustrated in Figure 2, comparison of the observed and calculated signal intensities resulted in a linear relationship with a slope of about 1.04 and a correlation coefficient of approximately 0.99 , indicating an additive, predictable signal. Successful analysis of mixed oligonucleotide sequences, modeled after DQB1 alleles frequently observed during genotyping of individuals from the CHP study of diabetics and their first-degree relatives, suggests that genotyping of heterozygous individuals is suitable to pyrosequencing methodology.

\section{Pyrosequence-Based Typing of HLA-DQB1 Allele *0301}

Genomic DNA containing the HLADQB 1 gene was prepared by PCR am plification using exon 2-specific primers DQBamp-A and DQBamp-B used in the 11th International Histocompatibility Workshop (8). DNA ob- tained from the HLA homozygous cell line BM16 (DQB1*0301) resulted in a single PCR product of the expected molecular weight when analyzed by gel electrophoresis (data not shown). How ever, the resulting pyrogram indicated the presence of at least two sequences (Figure 3A), as would be expected from a heterozygous source. For example, the anticipated sequence obtained from the HLA-DQB1*0301 allele using oligonucleotide primer PSQ1 is initially TTATGTGA, while the observed sequence exhibited a strong $\mathrm{G}$ signal at dispensation G4. Moreover, the signal intensities at nucleotide dispensations T1, A2, A11, A33, G34, and A35 are of one range, while the other dispensations exhibited much higher intensity, as though a second sequence was present and at a higher molar concentration.

Quantification of peak heights, followed by subtraction of the signal expected from the DQB $1 * 0301$ allele, resulted in the unmasking of a DNA sequence associated with a slope of roughly 0.99 and a correlation coefficient of approximately 0.92 , when the observed signal intensities were com pared to the calculated intensities for the DQB $1 * 0301$ sequence and the second sequence (Figure 3A). Identification of the DNA sequence was per-

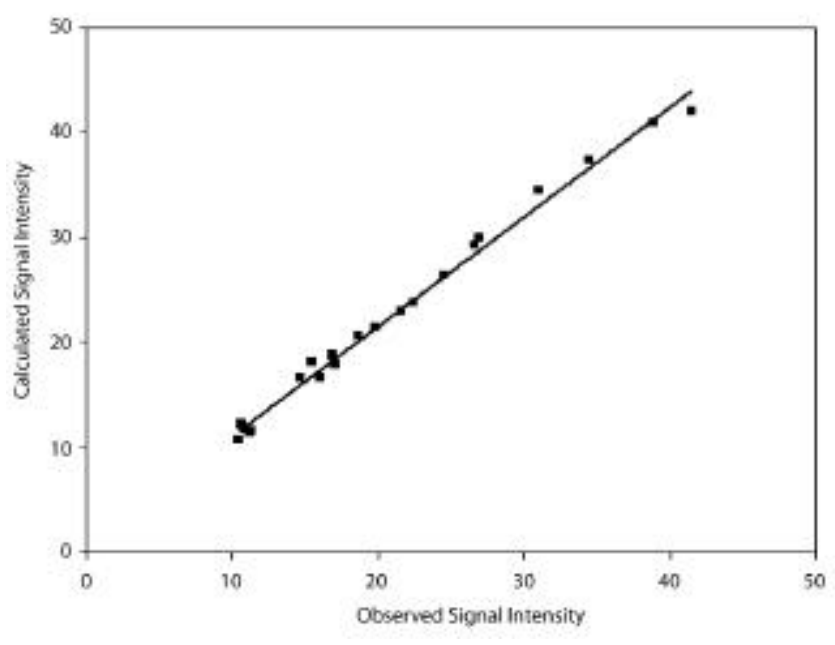

Figure 2. Plot of the observed signal intensity versus the calculated signal intensity for the oligonucleotide mixture of SR11 and SR14. Data were quantified from the pyrograms in Figure 1 using Peak Height Determination Software v1.1 and were background-corrected. Observed signal intensity was obtained using the peak height values from Figure $1 \mathrm{C}$, while calculated signal intensity was determined using peak height values from Figure 1, A and B, assuming an equal molar ratio of the two oligonucleotides. Calculated signal intensity for each peak is defined as $0.5 \times[$ (observed signal intensity for SR11) + (observed signal intensity for SR14)]. Linear regression analysis indicated a correlation coefficient of 0.99 and a slope and intercept of 1.04 and 0.71 , respectively. 
formed by BLAST analysis (1) against the publicly available human database, resulting in a complete match to a $27-$ nucleotide region of the HLA pseudogene DQB2 (Figure 3A). Moreover, BLAST analysis using the PCR primer sequences resulted in a complete match to regions of the pseudogene such that the expected PCR product would have a base-pair length identical to that obtained from amplification of DQB1 (data not shown). Identification of the combination of sequences DQB $1 * 0301$ and DQB2 indicated the usefulness of PSBT in HLA genotyping. However, the 11th International Histocompatibility Workshop primers, while previously considered sufficient for other genotyping technologies, are inappropriate for sequence-based typing methods requiring specific amplification of the DQB1 locus (17), possibly having contributed to ambiguous genotype analysis of HLA-DQB1.

Reengineered PCR primers SR22, SR24, and SR25 (Table 1) were synthesized following the recommendations of Pera et al. (17), with the intention of maximizing specific amplification of the DQB1 locus, but modified to avoid formation of self-priming 3' secondary structure that during pyrosequencing can lead to primer extension (24). PCR amplification of genomic DNA yielded a product of the expected molecular weight when analyzed by gel electrophoresis (data not shown). Pyrosequencing of the amplified DNA obtained from cell line BM16 (Figure 3B) resulted in a pyrogram for the expected DQB $1 * 0301$ allelic sequence, the result of a roughly $80 \%$ reduction in signal associated with the contaminating pseudogene (compare nucleotide dispensation G4 in Figure 3, A and B). Low-intensity signals at dispensations G4, C19, and G22 may indicate the presence of an additional contaminating PCR product, suggesting that further optimization of the PCR amplification step may result in improved data for pyrosequence-based typing.

\section{Pyrosequence-Based Typing of Genomic DNA from the CHP Study Samples}

Pyrosequence-based typing was performed, using DNA extracted from pe- ripheral blood mononuclear cells of diabetic patients and their immediate family members, on allelic combinations frequently observed in the CHP study population (16). Figure 4 shows data representing a 70-nucleotide length read of exon 2 of the HLADQB1 gene for a HLA-DQB1*0302 homozygous individual (Figure 4A) and for a HLA-DQB $1 * 0201-02+* 0302$ heterozygous individual (Figure 4B). Pyrograms exhibited the expected peak intensities for the homozygous and heterozygous combination (Figure 4). Amplified genomic DNA from the homozygous individual (Figure 4A) exhibited peak intensities corresponding to the expected sequence for the $* 0302$ allele; variations in signal intensity correlated to the number of nucleotide residues incorporated.

Pyrosequencing exhibited a drop in overall signal during the complete 69nucleotide dispensation cycle, which is probably the result of loss of apyrase activity (10). For the heterozygous individual (Figure 4B), signal intensities also correlated to the number of nucleotide residues incorporated, although for the combined *0201-02+ *0302 sequence. Moderately intense signal at nucleotide dispensations A29 and A39 (Figure 4B) did not correspond to the expected allelic combination, suggesting the presence of a contaminating sequence, indicating that additional optimization of PCR conditions may become necessary to improve the accuracy of pyrosequence-based typing. Complete pyrosequencing of the 270bp DQB1 gene exon 2 was performed using four sequencing primers (Table 1) and resulted in overlapping pyrosequence information (data not shown).

Pyrosequencing of genomic DNA obtained from individuals containing seven of the most common DQB1 allelic combinations (including the diabetogenic HLA-DQB $1 * 0302$ allele in com bination with alleles *0201-02,*0301, $* 0302$, or $* 0501)$ are summarized in Table 2. Genotyping was performed on samples from 49 individuals containing alleles accounting for greater than $80 \%$ of the total alleles observed in type $1 \mathrm{di}-$ abetics and greater than $70 \%$ of control

A

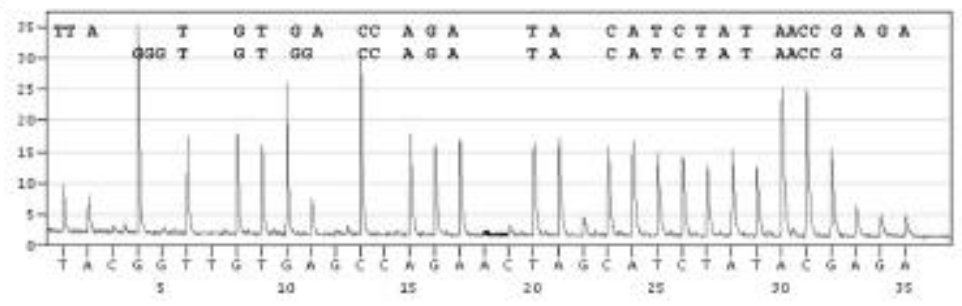

B

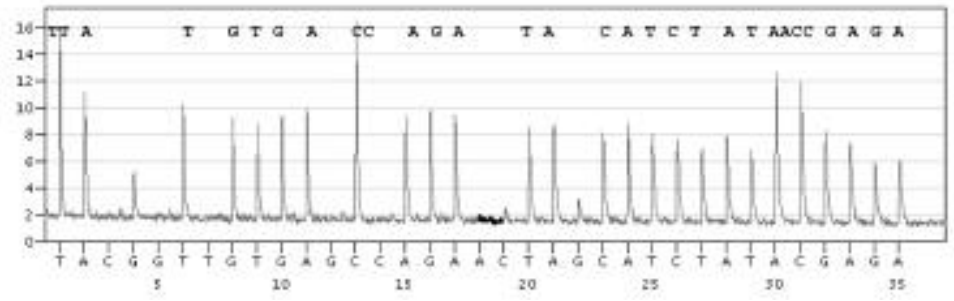

Figure 3. Pyrograms of HLA-DQB1 allele *0301 using 11th International Histocompatibility Workshop-recommended PCR primers DQBamp-A and DQBamp-B (A) or DQB1-specific PCR primers SR24 and SR25 (B). Pyrosequencing was performed on PCR-amplified DNA extracted from the HLA-DQB1*0301 homozygous cell line BM16 using DQB1-specific primers prepared at the 11th International Histocompatibility Workshop (DQBamp-A and DQBamp-B) (A) or primers engineered for this study (SR24 and SR25) based on the recommendations of Pera et al. (17) (B). Sequencing primer was PSQ1. Nucleotide sequences are indicated in panel A for DQB $1 * 0302$ (top sequence) and the pseudogene DQB2 (bottom sequence). The nucleotide sequence of allele DQB1*0302 is indicated in panel B. Axes are nucleotide dispensation event (x-axis) versus observed signal intensity ( $\mathrm{y}$-axis). 


\section{Molecular Diagnostic Techniques}

Table 2. Common DQB1 Alleles and Their Identity in Diabetic Families Sequenced in This Study

\begin{tabular}{|ccc|}
\hline $\begin{array}{c}\text { Number } \\
\text { of Samples }\end{array}$ & $\begin{array}{c}\text { SSOP-Identified } \\
\text { Alleles }\end{array}$ & $\begin{array}{c}\text { PSBT-Identified } \\
\text { Alleles }\end{array}$ \\
\hline $\begin{array}{c}\text { Heterozygous } \\
6\end{array}$ & ${ }^{*} 0201-02+{ }^{*} 0201-03$ & \\
14 & ${ }^{*} 0201-02+{ }^{*} 0302 / 07$ & ${ }^{*} 0201-02+{ }^{*} 0203$ \\
6 & ${ }^{*} 0201-02+{ }^{*} 0602 / 111$ & ${ }^{*} 0201-02+{ }^{*} 0302$ \\
6 & ${ }^{*} 03011 / 012+{ }^{*} 0302$ & ${ }^{*} 0301-02+{ }^{*} 0602$ \\
7 & ${ }^{*} 0302 / 07+{ }^{*} 05011 / 012$ & ${ }^{*} 0302$ \\
4 & ${ }^{*} 05011 / 012+{ }^{*} 0602 / 111$ & ${ }^{*} 0302+{ }^{*} 05011$ \\
Homozygous & ${ }^{*} 0302 / 07$ & ${ }^{*} 0602$ \\
6 & ${ }^{*} 0302$ \\
aSource of sequence-specific oligonucleotide probe (SSOP) genotyping: CHP \\
Histocompatibility Center study of diabetic patients and their immediate family \\
members. Ambiguously SSOP-typed alleles are indicted in bold. \\
bEach SSOP-identified subpopulation examined in this study resulted in an iden- \\
tical pyrosequence-based typing (PSBT)-identified genotype. Alleles resolved \\
by PSBT are indicated in bold.
\end{tabular}

subjects from the CHP study population (5). Pyrograms readily distinguished these allelic combinations, leading to their immediate identification as well as being able to resolve ambiguous results obtained from sequence-specific oligonucleotide probe-based typing (Figure 4 and Table 2). An apparent advantage of pyrosequence-based typing, in contrast to hybridization-based methods, is that an oligonucleotide primer initiates the sequencing of a 70-nucleotide region of the allele, while hybridization by oligonucleotide probe identifies only the complementary annealing site. Predictions of the number of oligonucleotide probes required to identify the DQB1 allelic combinations included a high number necessary to distinguish 18 individual codon positions (14), which is a number of primers similarly required for analysis by mass spectrophotometry-based methods. Pyrosequence-based typing required fewer primers for genotyping of the highly polymorphic HLA locus than hybridization-based methodologies.

Simulation of pyrosequencing data was performed for the complete set of DQB1 alleles, using a computer algorithm to generate the predicted pyrograms for each allelic combination. Identification of unique pyrograms was performed by comparing the theoretical results of each possible allelic combination (data not shown). The 44 unique DQB1 exon 2 sequences resulted in 990 potential allelic pairs, of which 982

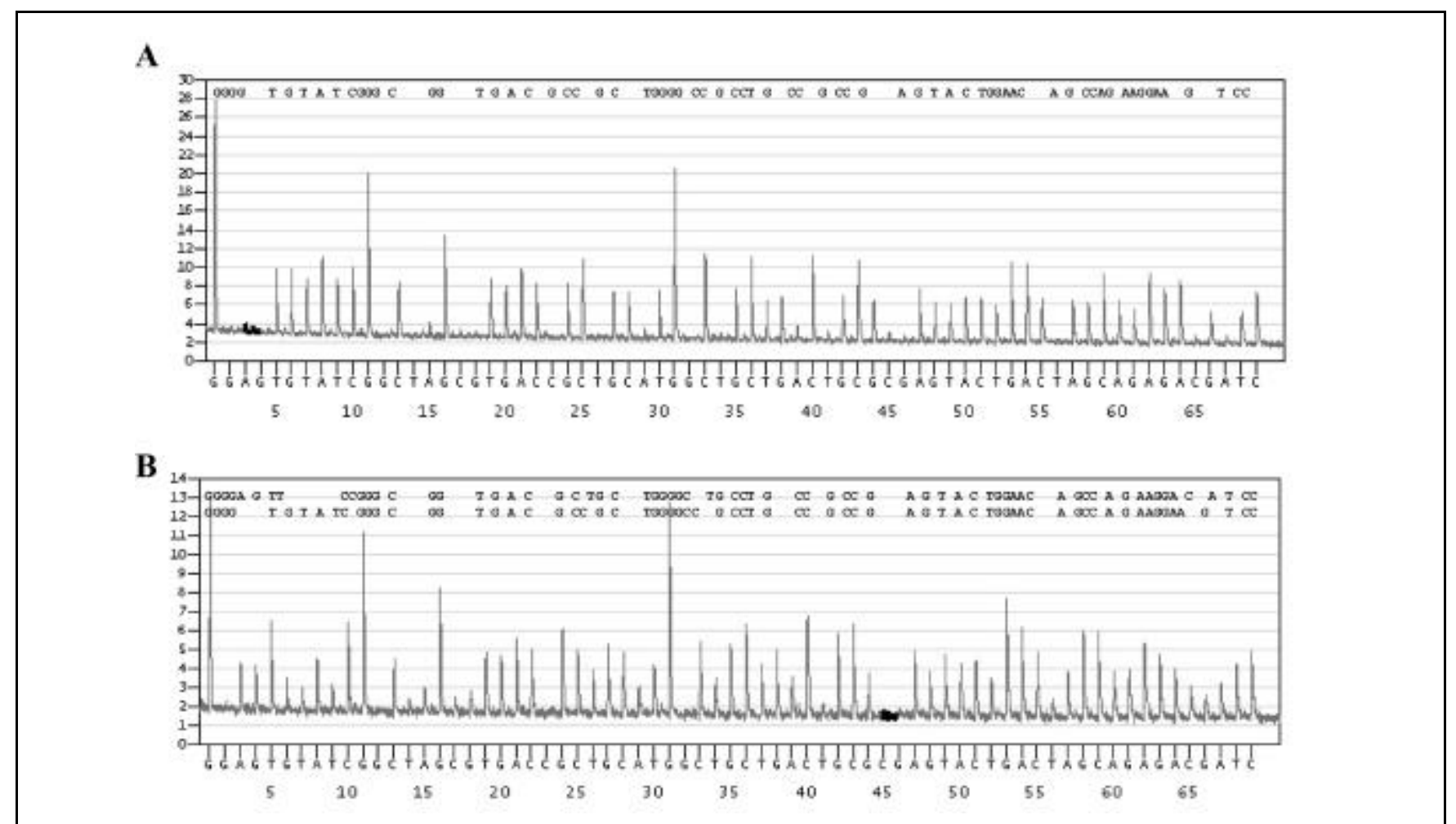

Figure 4. Pyrograms of HLA-DQB1 alleles prepared from peripheral blood mononuclear cells extracted genomic DNA samples from the CHP study of diabetics and their immediate family members. Pyrograms illustrate sequence data from a homozygous *0302 individual (A) and a heterozygous *201$02+* 0302$ individual (B). Nucleotide sequences are indicated for *0302 in panel A and for *0201-02 (top sequence) and *0302 (bottom sequence) in panel B. Read lengths were at least 70 nucleotides. Sequencing primer was PSQ3. Axes are nucleotide dispensation event (x-axis) versus observed signal intensity (y-axis). 


\section{M olecular Diagnostic Techniques}

gave unique pyrograms, a predicted success rate of roughly $99 \%$. Eight allelic pairs yielded identical pyrograms and occurred in four sets: $(i) * 0201-$ $02+* 03011 / 09$ or $* 0203+* 0304$; (ii) *0201-02+*03032 or *0203+*0302; (iii) $* 03011 / 09+* 0302$ or $* 03032+$ $* 0304$; and (iv) *05012+*0610 or $* 0502+* 0613$. Changes to the pyrosequencing protocol (e.g., the order of nucleotide dispensation) may further optimize the ability to distinguish between the remaining allelic combinations (unpublished data). Amplification of DQB1 exons other than exon 2 will be required to distinguish among alleles $* 0201 / * 0202$, *03011/*0309, and *06011/*06013. A similar analysis, based on methods used in sequencebased typing, has been posted at the IMGT/HLA database (http://www.ebi. ac.uk/imgt/hla/) $(20,21)$. In contrast to pyrosequence-based typing, sequencebased typing was predicted to result in
26 ambiguous allelic pairs when the DQB1 gene was examined.

\section{DISCUSSION}

Pyrosequencing methodology represents an improvement in automation, including the advantages of real-time data output, absence of sequencing artifacts associated with gel electrophoresis, no need for DNA fluorescent labeling, and compatibility with 96- and 384-well microplate formats. Originally formulated for sequencing short stretches of DNA, pyrosequencing has been evaluated for incorporation into genomic typing protocols with the goal of improving throughput and accuracy of HLA genotyping.

Pyrosequence-based typing of HLA alleles was performed on prevalent allelic combinations observed in diabetics and their first-degree relatives. Py- rograms confirmed the assignment of alleles obtained by sequence-specific oligonucleotide probe-based typing and provided unambiguous additional resolution. Complete sequencing of the highly polymorphic exon 2 of HLADQB1 was performed in four reactions, resulting in overlapping sequence information. Different reaction conditions may allow the sequencing of a longer stretch of DNA (10), further reducing the number of overlapping segments needed. Although the polymorphisms at some other HLA loci will result in a more complex analysis, particularly HLA class I loci, the results obtained by sequencing DQB1 alleles can be considered the first step to expand pyrosequencing applications to a complete HLA typing.

Optimization of the pyrosequencebased typing protocol allowed the rapid identification of alleles for the analysis of genetic predisposition to disease and 
for histocompatibility typing. Considering that a single pyrosequencing instrument is capable of performing sequencing of a 96-well tray every hour, up to 15000 sequence reactions can be analyzed within a month. On this basis, pyrosequencing methodology seems to possess all the attributes to enhance the throughput and specificity of HLA typing via the rapid acquisition of DNA sequence information.

\section{ACKNOWLEDGMENTS}

We thank Jennifer Profozich and Therese Libert for synthesis of oligonucleotides, Lynn Nichol for providing purified genomic DNA, and Patrick Hnidka for final preparation of the manuscript. This work was supported by National Institutes of Health grant no. RO1DK24021, ERHS \#00035010 from the Department of Defense, and N00014-01-1-0839 from the Office of Naval Research to M.T.

\section{REFERENCES}

1.Altschul, S.F., T.L. Madden, A.A. Schaffer, J. Zhang, Z. Zhang, W. Miller, and D.J. Lipman. 1997. Gapped BLAST and PSIBLAST: a new generation of protein database search programs. Nucleic Acids Res. 25:33893402.

2.Boehm, B.O., B. Manfras, S. Seidl, G. Holzberger, P. Kuhnl, C. Rosak, K. Schoffling, and M. Trucco. 1991. The HLA-DQ beta non-Asp-57 allele: a predictor of future insulin-dependent diabetes mellitus in patients with autoimmune Addison's disease. Tissue Antigens 37:130-132.

3.Cavender, D.E., D.K. Wagener, B.S. Rabin, D.J. Becker, T.J. Orchard, M.S. Eberhardt, R.E. LaPorte, A.L. Drash, and L.H. Kuller. 1984. The Pittsburgh Insulin-Dependent Diabetes Mellitus (IDDM) study. HLA antigens and haplotypes as risk factors for the development of IDDM in IDDM patients and their siblings. J. Chronic Dis. 37:555-568.

4.Davies, J.L., Y. Kawaguchi, S.T. Bennett, J.B. Copeman, H.J. Cordell, L.E. Pritchard, P.W. Reed, S.C. Gough, et al. 1994. A genome-wide search for human type 1 diabetes susceptibility genes. Nature 371:130136.

5.Dorman, J.S., R.E. LaPorte, R.A. Stone, and M. Trucco. 1990. Worldwide differences in the incidence of type I diabetes are associated with amino acid variation at position 57 of the HLA-DQ beta chain. Proc. Natl. Acad. Sci. USA 87:7370-7374.

6.Drash, A.L., R.B. Lipton, J.S. Dorman, D.J. Becker, R.E. LaPorte, T.J. Orchard, W.J.
Riley, M. Trucco, and L.H. Kuller. 1991 The interface between epidemiology and molecular biology in the search for the causes of insulin-dependent diabetes mellitus. Ann. Med. 23:463-471.

7.Erlich, H.A., G. Opelz, and J. Hansen. 2001 HLA DNA typing and transplantation. Immunity $14: 347-356$.

8.Faas, S.J., R. Menon, E.R. Braun, W.A. Rudert, and M. Trucco. 1996. Sequence-specific priming and exonuclease-released fluorescence detection of HLA-DQB1 alleles. Tissue Antigens 48:97-112.

9.Friday, R.P., M. Trucco, and M. Pietropaolo. 1999. Genetics of Type 1 diabetes mellitus. Diabetes Nutr. Metab. 12:3-26.

10.Gharizadeh, B., T. Nordstrom, A. Ahmadian, M. Ronaghi, and P. Nyren. 2002. Longread pyrosequencing using pure 2'-deoxyadenosine-5'-O'-(1-thiotriphosphate) Sp-isomer. Anal. Biochem. 301:82-90.

11.Hsia, S., J.Y. Tong, G.L. Parris, D.D. Nghiem, E.M. Cottington, W.A. Rudert, and M. Trucco. 1993. Molecular compatibility and renal graft survival - the HLA DRB1 genotyping. Transplantation 55:395-399.

12.Hurley, C.K., M. Maiers, J. Ng, D. Wagage, J. Hegland, J. Baisch, R. Endres, M. Fernandez-Vina, et al. 2000. Large-scale DNAbased typing of HLA-A and HLA-B at low resolution is highly accurate specific and reliable. Tissue Antigens 55:352-358.

13.LaPorte, R.E., A.L. Drash, D. Wagener, T.J. Orchard, and L.H. Kuller. 1982. The Pittsburgh insulin-dependent diabetes mellitus (IDDM) registries: the descriptive epidemiology of racial differences, p. 66-77. In $\mathrm{G}$ Mimura, S. Baba, Y. Goto, and J. Kobberling (Eds.), Clinico-Genetic Genesis of Diabetes Mellitus. International Congress Series 597. Excerpta Medica, Amsterdam, The Netherlands.

14.Luo, M., J. Blanchard, Y. Pan, K. Brunham, and R.C. Brunham. 1999. High-resolution sequence typing of HLA-DQA1 and DQB1 exon 2 DNA with taxonomy-based sequence analysis (TBSA) allele assignment. Tissue Antigens 54:69-82

15.McDevitt, H. 2001. Closing in on type 1 diabetes. N. Engl. J. Med. 345:1060-1061

16.Morel, P.A., J.S. Dorman, J.A. Todd, H.O. McDevitt, and M. Trucco. 1988. Aspartic acid at position 57 of the HLA-DQ $\beta$ chain protects against type I diabetes: a family study. Proc. Natl. Acad. Sci. USA 85:8111-8115.

17.Pera, C., L. Delfino, A. Longo, M.P. Pistillo, and G.B. Ferrara. 2000. Novel associations among HLA-DQA1 and -DQB1 alleles, revealed by high-resolution sequence-based typing (SBT). Tissue Antigens 55:275-279.

18.Pietropaolo, M., D.J. Becker, R.E. LaPorte, J.S. Dorman, S. Riboni, W.A. Rudert, S. Mazumdar, and M. Trocco. 2002. Progression to insulin-requiring diabetes in seronegative prediabetic subjects: the role of two HLADQ high-risk haplotypes. Diabetologia 45:66-76.

19.Pietropaolo, M. and M. Trucco. 2000. Major histocompatibility locus and other genes that determine the risk of development of type 1 diabetes mellitus, p. 399-410. In D. LeRoith, S.I. Taylor, and J.M. Olefsky (Eds.), Diabetes
Mellitus: A Fundamental and Clinical Text, 2nd ed. Lippincott Williams and Wilkins, Philadelphia, PA.

20.Robinson, J., A. Malik, P. Parham, J.G. Bodmer, and S.G.E. Marsh. 2000. IMGT/ HLA database - a sequence database for the human major histocompatibility complex. Tissue Antigens 55:280-287.

21.Robinson, J., M.J. Waller, P. Parham, J.G. Bodmer, and S.G.E. Marsh. 2001. IMGT/HLA Database-a sequence database for the human major histocompatibility com plex. Nucleic Acids Res. 29:210-213.

22.Ronaghi, M. 2001. Pyrosequencing sheds light on DNA sequencing. Genome Res. 11:311 .

23.Ronaghi, M., S. Karamohamed, B. Pettersson, M. Uhlen, and P. Nyren. 1996. Realtime DNA sequencing using detection of pyrophosphate release. Anal. Biochem. 242:84-89

24.Ronaghi, M., B. Pettersson, M. Uhlën, and P. Nyrën. 1998. PCR-introduced loop structure as primer in DNA sequencing. BioTechniques 25:876-878.

25.Ronaghi, M., M. Uhlen, and P. Nyren. 1998 A sequencing method based on real-time pyrophosphate. Science 281:363-365.

26.Rosner, G., B.S. Martell, and M. Trucco. 2000. Histocompatibility, p. 233-251. In E.D. Ball, J. Lister, and P. Law (Eds.), Hematopoietic Stem Cell Therapy. Churchill Linvingstone, Philadelphia, PA.

27.Todd, J.A., J.I. Bell, and H.O. McDevitt. 1987. HLA-DQ $\beta$ gene contributes to susceptibility and resistance to insulin-dependent diabetes mellitus. Nature 329:599-604.

28.Tong, J.Y., S. Hsia, G.L. Parris, D.D. Nghiem, E.M. Cottington, W.A. Rudert, and M. Trucco. 1993. Molecular compatibility and renal graft survival- the HLA DQB1 genotyping. Transplantation 55:390-395.

29.Trucco, M. 1992. To be or not to be Asp 57, that is the question. Diabetes Care 15:705715

Received 20 February 2002; accepted 22 April 2002.

Address correspondence to:

Dr. Massimo Trucco

Division of Immunogenetics

Rangos Research Center

Children's Hospital of Pittsburgh

3460 Fifth Avenue

Pittsburgh, PA 15213, USA

e-mail:mnt@pitt.edu
For reprints of this or any other article, contact Reprints@BioTechniques.com 